\title{
LXI. \\ Ueber die unorganischen Bestandtheile der Ranken, Blätter, Trauben und Kerne des Weinstocks.
}

\author{
Von \\ P. Berthier.
}

(Annal. de chim. at de phys. Norbr. 1851, 210).

Der 2u dieser Untersuchung dienende Weinstock (gamay) war sechs bis sieben Jahre alt und an einem sandigen Ufer bei Nemours, am Zusammenfluss des Kanals und der Loing, cullivirt. Dieses sandige Ufer war fruchtbar, da sein Unterboden, wenigstens bei 1 Meter Tiefe, mit kalkhaltigen Quellen bewissert war.

Der Weinstock war aus einem in sehr gutem Zustande befindlichen Weinlıerge, welcher zum Dünger lauptsüchlich alles das erhailt, was der Weinberg selbst giebt (Ilolz, Blätter etc.), nebst dünnen Zweigen von IIolz und Kiäutern, die das Verschneiden einer IIccke und ier Garteneinfassungen liefert.

Der Weinstock war ganz gesund und mit reifen Trauben reichlich beladen. $\mathrm{Er}$ wurde im October 1850 zur Weinlese ganz abgeschnitten. Die Trauben wurden besonders gelegt und auch die Ranken mit iliren noch grünen Blältern, denen man noch alles Holz und die Blätter zufügte, welche beim Schneiden im Sominer erhalten worden waren. Das Ganze wurde während fünf Monaten in einem verschlossenen Zimmer getrocknet.

Holiz und Blätter. Im März wogen die Ranken und Blätter zusammen $\mathbf{4 5 0}$ Grammen. Sie wurden mit Leichtigkeit verbrannt und lieferten 29,50 Grm. einer weissen Asche. Da diese aber mit einer ziemlich grossen Henge Sand vermischt war, welcher wahrscheinlich den Blättern anhaftete, und dagegen eine gevvisse Menge Kohlensäure fehlte, die zur Săttigung des Kalks nothwendig war, so beträgt das Gewicht der reinen Asche nur 26,50 Grm. $=0,059$.

Diese Asche mit heissem Wasser gewaschen, lieferte:

$\begin{array}{lrr}\text { Loslicher Theil (geschmolzue alkalische Salze) } & \begin{array}{r}\text { Grm. } \\ 6,20\end{array} & \mathbf{0 , 2 3 4} \\ \text { Unloslicher Theil (erdige Salze) } & \frac{20,30}{26,30} & \frac{0,766}{1,008}\end{array}$ 
folglich liefert 1,0000 Rebe:

$$
\begin{array}{ll}
\text { Alkalische Salze } & \mathbf{0 , 0 1 3 8} \\
\text { Erdige Salze } & \mathbf{0 , 0 6 5 2}
\end{array} \quad \mathbf{0 , 0 5 9 0}
$$

Der lösliche Theil der Asche wurde zusammengesetzt gefunden aus :

\begin{tabular}{lll} 
Schwefelsantem Kali & $\mathbf{G r m .}$ & \\
Chlorkalium & $\mathbf{1 , 1 7 0}$ & $\mathbf{0 , 1 8 8}$ \\
Kohlensaurc Alkalien & 0,600 & $\mathbf{0 , 0 9 6}$ \\
Gallertartige Kieselsāure & $\mathbf{4 , 2 8 0}$ & $\mathbf{0 , 0 0 9 2}$ \\
\cline { 2 - 2 } & $\mathbf{0 , 1 5 0}$ & $\mathbf{0 , 0 2 4}$ \\
\cline { 2 - 3 } & $\mathbf{6 , 2 0 0}$ & $\mathbf{1 , 0 0 0}$
\end{tabular}

Die Analyse der unlöslichen Theile ergab:

\begin{tabular}{lrr} 
Kohlensauren Kalk & Grm. & \\
Kohlensaure Magnesia & $\mathbf{1 3 , 1 7 0}$ & $\mathbf{0 , 6 4 9}$ \\
Phosphorsauren Kalk & $\mathbf{1 , 0 2 0}$ & $\mathbf{0 , 0 5 0}$ \\
Phosphorsaures Eisenoxyd & $\mathbf{4 , 1 5 0}$ & $\mathbf{0 , 2 0 6}$ \\
Gallerartige Kieselsäure & $\mathbf{0 , 3 1 0}$ & $\mathbf{0 , 0 2 5}$ \\
& $\mathbf{1 , 4 2 0}$ & $\mathbf{0 , 0 7 0}$ \\
\hline $\mathbf{2 0 , 3 0 0}$ & $\mathbf{1 , 0 0 0}$
\end{tabular}

Die ganze Asche enthielt demnach:

$\begin{array}{ll}\text { Schwefelsaures Kali } & 0,0440 \\ \text { Chlorkanlium } & 0,0220 \\ \text { Kohlcusaure Alkalien } & 0,1640\end{array}$ Alkalische Salze 0,2300

Trauben. Die Weintrauben wurden auf einem Teller getrocknet, und wogen dann 70 Grammen. Sie hinterliessen nach dem Glühen in einem bedeckten Tiegel 15 Grammen Kohle $(=0,21)$ und diese gab verbrannt $2,96 \mathrm{Grm}$. einer grauweissen Asche $(=0,042)$. Diese 'Asche wurde zusammengesetzt gefunden aus:

Schwefelsaurem Bali

Chlorkalium

Kohlensauren Alkalien

Kohlensaurem Kalk

Kohlensaurer Magnesia

Phosphorsaurem Ralk

Kieselsăure

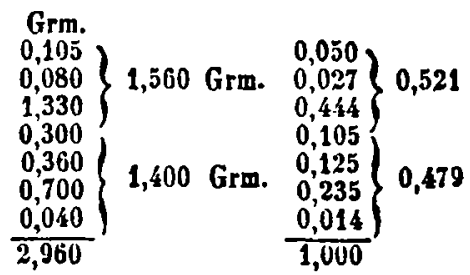

Diese verschiedenen Resultate zusammengestellt ergeben:

1) dass der trockne Stock $450 \mathrm{Grm}$. und die Trauben 70 " wogen. 
2) dass der Stock Asche

$26,50 \mathrm{Grm} .=0,0590$ lieferte, welche enthielt:
Alkalische Salze
$6,20 \mathrm{Grm} .=0,0138$
Erdige Salze
$20,30,=0,0452$

3) während die Trauben

Asche

2,96 Grm. $=0,0420$ liefer-

ten, welche enthielten:

Alkalische Salze $\quad 1,56 \mathrm{Grm} .=0,0225$

Erdige Salze $\quad 1,40, \quad=0,0195$

4) und dass demnach der Weinstock enthielt:

Neunmal soviel unorganische Stoffe als die Weintrauben

\section{Viermal soviel alkalische Salze}

Vierzehnmal soviel erdige Salze, unter andern

Sechs bis siebenmal soviel phosphorsaure.

Es geht hieraus hervor, dass die alkalischen Substanzen zum Wachsthum des Ilolzes und der Blätter nülhiger sind als zur Production der Trauben, und dass diese, eben so wie das IIolz, phosphorsauren Kalk nüthig haben. Die Rathscbläge, welche man gegeben hat, die jungen, noch nicht tragbaren Weinstücke vorzüglich mit phosphorsauren Salzen zu düngen, und die alkalischen Düngungsmittel für die fruchttragenden Weinstöcke zu verwenden, stimmen demnach nicht mit den beobachteten Thatsachen überein.

Wenn man die Analysen, welche mit verschiedenen Weinen angestellt wurden, in Erwägung zieht, so kann der geklärte Traubensaft nur eine geringe Menge unorganischer Stoffe enthalten (Tartrate des Kalis und des Kalks), es würde demnach die Asche der Trauben grösstentheils von den Schalen, den Gefāss - und Zellen-Substanzen und von den Kernen herrühren, welche Stoffe als Trester bei der Weinfabrication zurückbleiben. Die Menge der alkalischen Aschen-Substanzen, welche der Wein enthält, betrăgt demnach nur den geringsten Theil der Gesamnimenge, welche die Cultur dem Boden entzieht, und kann für dieselbe niclit als Maassstab dienen. Die am meisten erschípfenden Weinstöcke sind daher nicht diejenigen, welche am productivsten sind, sondern diejenigen, welche kräflig regetiren und ein grosses Volumen Bolz und Blätter erzeugen, um so mebr da die 
Weintrester fast beständig durch den Dünger wieder in den Boden zurückkehren. Ein Weinberg von einer Hectare, der 10000 den analysirten ähnliche Weinstöcke enthielte, würde dem Boden jährlich ohngefähr 75 Kilogrammen alkalischer Salze entziehen, nämlich 60 Kilogrm. für die Reben und Blătter und 15 Kilogrm. für die Früclte.

Kerne rother Trauben. Ich verschaffte mir sorgfältig von Parenchym befreite Kerne dadurch, dass die aus der Presse kommenden Weintrester mit einer grossen Menge Wasser umgerührt und die $\mathrm{zu}$ Boden fallenden Kerne gesammelt wurden. Sie wurden vollends durch Reiben mit den Händen unter fliessendem Wasser gereinigt und dann in einem verschlossenen Zimmer während mehrerer Monate der freiwilligen Austrocknung überlassen. Sie gaben durch Glühen in einem verschlossenen Gefässe 0,214 Kohle, und nach der Verbrennung 0,020 weisse Asche. Die Analyse dieser Asche ergab:

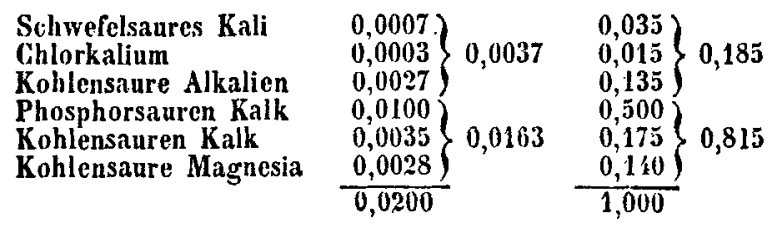

Da diese Samen sehr klein und hart sind, so muss die holzige Hülle einen beträchtlichen Theil ihres Gewichts ausmachen; daraus folgt, dass der innere Kern eine sehr grosse Menge phosphorsauren Kalk enthält, denn die Asche des Holzes besteht bekanntlich aus Carbonaten des Kalis und des Kalks.

Blätter. Wie wir wissen sind die Blätter diejenigen Theile der Gewächse, welche die meiste Asche liefern. Ich habe diese Thatsache beim Weinstock bestätigt gefunden, indem ich eben so viele grünende als abgestorbene Blätter analysirte.

Grïnende Blätter. Die grünenden Blătter wurden in ihrer grōssten Entwickelung im Juli gesammelt. $2000 \mathrm{Grm}$. derselben wurden auf dem Boden einer verschlossenen Kammer unter ofterem Umwenden sechs Wochen lang aufbewahrt. Sie hinterliessen 500 Grammen $(=0,250$ ). Sie wurden, obne vollkommen trocken zu sein, mit Leichtigkeit verbrannt und gaben sandfrei berechnet $42 \mathrm{Grm}$. Asche, oder 0,021 aus den grünen 
und 0,084 aus den trocknen Blättern. Diese Asche war zusammengesetzt aus :

$\left.\begin{array}{ll}\text { Schwefelsaurem Kali } & 0,070 \\ \text { Chlorkalium } & 0,008 \\ \text { Kohlensauren Alkalien } & 0,072 \\ \text { Kohlensaurem Kalk } & 0,510 \\ \text { Kohlensaurer Magnesia } & 0,034 \\ \text { Phosphorsaurem Kalk } & 0,153 \\ \text { Phosphorsaurem Eisenoxyd } & 0,051 \\ \text { Kieselsaiure } & 0,102\end{array}\right\}$ Erdige Salise 0,850

Die grünen Blätter enthielten demnach 0,0031 alkalische Salze und die trocknen Blătter wenigstens 0,0126.

Abgestorbene Blätter. Die abgestorbenen Blätter wurden von den nämlichen Weinstücken gesammelt, wenn sie abfallen wollten, ihre grüne Farbe jedoch nicht verloren hatten. $1500 \mathrm{Grm}$. wurden zwei Monate lang zum Trocknen in einer verschlossenen Kammer aufbewahrt, und wogen nachlier $500 \mathrm{Grm}$. $(=0,33)$. Sie hatten noch eine deutlich grüne Farbe, liessen sich mit den Fingern leicht zerreiben und verbrannten selır schnell. Sie hinterliessen $56,70 \mathrm{Grm}$. als rein berechnete Asche $(=0,1134)$ und enthiclten:

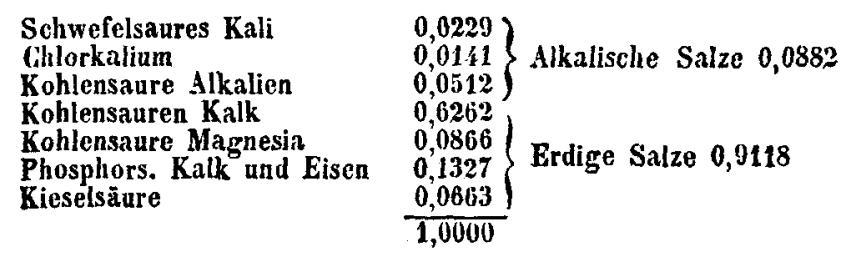

Dic rohen Blätter enthielten demnach 0,0033 alkalische Salze, die getrockneten $\mathbf{0 , 0 1 0}$.

Die Mengen der unorganischen Bestandtheile, welche in dem beblätterten Holze, in den grünenden und abgestorbenen Blăttern, alle an der Luft getrocknet, enthalten sind, betragen demnach:

: : 0,0059 (Holz) : 0,084 (grünende Blătter) und 0,1134 (abgestorbene Blătter), die Mengen der alkalischen Substanzen:

: : 0,2138 (Holz) : 00126 (grünende Bl.) und 0,0100 (abgestorbene $\mathrm{Bl}$. ), und die Mengen der erdigen Salze: 
: : 0,0452 (Holz) : 0,0714 (grünende Bl.) und 0,1034 (abgestorbene $\mathrm{Bl}$.).

Das nackte Holz musste demnach ohngefähr denselben Gehalt an Alkalien haben, als die grünenden Blätter; leider wurde diess nicht durch die Analyse năher bestimmt.

Aus diesen Resultaten folgt, dass die anorganischen Stoffe des Weinstocks, eben so wie bei den meisten Gewächsen, in viel grŏsseren Mengen in den Blättern als im Holze enthalten sinl. Die Aschen der Blätter enthalten aber viel weniger Alkalien als die des Holzes. Nichts destoweniger finden sich die alkalischen Stoffe fast gleichmässig in den verschiedenen Pflanzentheilen vertheilt.

Der in die Blätter aufsteigende Saft, verdunstet beträchtlich in ihnen, und man kann annehmen, dass er sich in Folge der Verdunstung und erfolgender chenischer Veränderungen des grüssten Theils der erdigen Substanzen entledigt, welche er in Aullősung hielt, während der absteigende Saft in den Körper der Pflanzen die noch löslichen Salze zurückfülurt, unter ihnen einen grossen Theil der alkalischen Salze.

$$
Z \text { u } s \text { a } t \text { : }
$$

Analyse der frischen Traube. Zur vollständigen Aufklärung dieses Gegenstandes, untersuchte ich die unorganischen Substanzen in den verschiedenen Theilen der frischgesammelten Trauben.

Ich untersuchte zwei Traubensorten: 1) weissen Gutedel (chasselas blanc) aus der Umgegend von Paris, und 2) eine schwarze Traube, (pineau) ebenfalls aus der Umgegend von l'aris. Die Trauben wurden abgebeert, und die Stiele gewogen bei Seite gelegt. Die Beeren wurden zwischen den Händen zerdrückt, alsdann so gut als möglich ohne eine Presse in einem Tuche ausgepresst, so dass die Trestern noch sehr feucht blieben. Diese wurden gewogen, aus der Differenz ergab sich das Gewicht des Saftes. Da letzterer etwas trübe war, wurde er filtrirt und vollkommen klar erhalten. Er war hell, nahm aber bei der Berührung mit der Iuft eine etwas braungelbe Farbe an. Beim Verdampfen wurde sie dunkler und ging in schwarzbraun über. Der Syrup nahm die Consistenz der Melasse an, blähte sich auf, und wurde endlich so brennbar, dass er 
sich auf dem Boden der Schale entzündete, während noch der obere Theil weich war. Das Ganze wurde in einer Platinschale vollkommen . eingeåschert. Die Stiele und die Trestern wurden eben so behandelt. Endlich wurde die Analyse der drei Aschen gemacht, mit vorzüglicher Berücksichtigung der relativen Menge der alkalischen Salze und der erdigen Substanzen. Folgendes sind die Resultate:

Weisser Gutedel:

$\left.\begin{array}{ll}\text { Stiele } & \mathbf{0 , 0 4 2} \\
\text { Trester } & 0,220 \\
\text { Filtrirter Saft } & 0,738\end{array}\right\}$ Asche \begin{tabular}{l}
0,00060 \\
0,00110 \\
\cline { 2 - 3 }
\end{tabular}

Also erzengten:

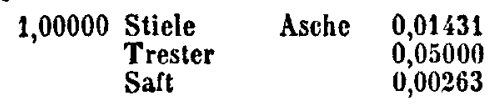

Die ganze Weintraube besteht folglich aus:

\begin{tabular}{lccc} 
& Stiele. & Trester. & Saft. \\
Alkalischen Salzen & 0,00020 & 0,00060 & 0,00100 \\
Phosphorsaurem Kalk & $\mathbf{0 , 0 0 0 1 1}$ & $\mathbf{0 , 0 0 0 3 0}$ & 0,00047 \\
Kohlensaurem Kalk & $\mathbf{0 , 0 0 0 2 0}$ & $\mathbf{0 , 0 0 0 1 2}$ & 0,00035 \\
Kohlensaurer Magnesia & - & 0,00008 & $\mathbf{0 , 0 0 0 1 2}$ \\
\cline { 2 - 4 } & $\overline{0,00060}$ & $\overline{0,00110}$ & $\mathbf{0 , 0 0 1 9 4}$
\end{tabular}

Diese Aschen wurden zusammengesetzt gefunden aus:

$$
\begin{aligned}
& \text { Alkalischen Salzen } \quad 0,00180 \quad 0,00180 \\
& \left.\begin{array}{ll}
\text { Phosphorsaurem Kalk } & \mathbf{0 , 0 0 0 9 1} \\
\text { Kohlensaurem Kalk } & \mathbf{0 , 0 0 0 7 3}
\end{array}\right\} \mathbf{0 , 0 0 1 8 4} \\
& \text { Kohlensaurer Magnesia } \frac{0,00020}{0,00364}
\end{aligned}
$$

Die alkalischen Salze bestehen wesentlich aus Carbonaten, aber sie enthalten ausserdem eine bemerkenswerthe Menge an Sulphat und eine Spur Chlorür, diejenigen der Trester enthalten ferner eine kleine Menge Phosphat. Der phosphorsaure Kalk war kaum gefärbt und enthieit nur eine Spur Eisenoxyd.

Schuarze Traube. Sie ergab:

$$
\begin{aligned}
& \text { Stiele } \quad 0,036) \quad 0,00060 \\
& \text { Trester } 0,240\} \text { Aschen } 0,00110 \\
& \text { Filtrirter Saft } 0,724 \text { As } 0,00298
\end{aligned}
$$

Folglich erzeugten:

$$
\begin{aligned}
& \begin{array}{ccc}
1,00000 & \text { Stiele } & \text { Aschen } \\
\text { Trester } & 0,01700 \\
0,04600
\end{array} \\
& \text { Saft } \quad 0,00400
\end{aligned}
$$


Diese Aschen enthielten:

\begin{tabular}{lccc} 
& Stiele & Trester. & Saft. \\
Alkalische Salze & $\mathbf{0 , 0 0 0 2 0}$ & 0,00060 & $\mathbf{0 , 0 0 1 5 k}$ \\
Phosphorsauren Kalk & 0,00014 & 0,00030 & 0,00072 \\
Kohlensauren Kalk u. Magnesia & $\mathbf{0 , 0 0 0 2 6}$ & $\mathbf{0 , 0 0 0 2 0}$ & $\mathbf{0 , 0 0 0 7 2}$ \\
\cline { 2 - 3 } & $\mathbf{0 , 0 0 0 6 0}$ & $\mathbf{0 , 0 0 1 1 0}$ & $\mathbf{0 , 0 0 2 9 8}$
\end{tabular}

Die ganze Traube enthielt folglich:

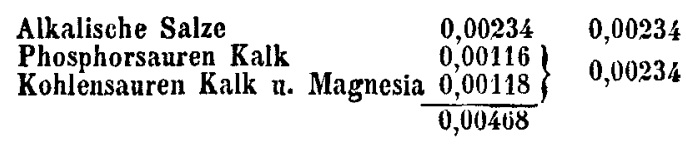

Für diese Aschen gelten übrigens dieselben Bemerkungen wie für die weissen Trauben.

Man sieht, dass diese beiden Trauben fast identische Resultate geliefert haben. Nur bat der Saft der schwarzen Traube etwas mehr Asche hinterlassen, als der der weissen Traube. Beide enthalten nur eine sehr geringe Menge von Alkalien. Dieses hat übrigens schon Bouchardat gefunden, welchem wir wichtige Arbeiten über die Cultur des Weinstocks und über die Fabrication der Weine verdanken. Bei der Analyse der filtrirten Săfte verschiedener Weinsorten, fand er niemals melır als 0,00067 Kali (wahrscheinlich als ätzendes und wasserfreies berechnet), er bemerkte, dass bisweilen das Verhältniss dieses Alkali bis zu 0,00045 herabsinke. Er untersuchte nicht die erdigen Bestandtheile, sagt aber, dass, wenn die Weine trinkbar geworden sind, sie fast keinen Kalk enthalten, und dass sie beim Verdampfen nur 0,022 trocknen Rückstand hinterlassen. Er hat diesen Rūckstand nicht geglüht um zu erfahren, wie viel er Asche hinterlasse.

Aus allen diesem geht hervor, dass weder der Wein noch die Traube dem Boden das Alkali entziehen, sondern dass das Holz und die Blätter den grōssten Theil davon absorbiren. 